\title{
Notice
}

\section{Corrigenda to "Local structure of feasible sets in nonlinear programming, Part III: Stability and sensitivity"}

by Stephen M. Robinson

Mathematical Programming Study 30 (1987) 45-66.

1. Eq. (1.8) requires a sign correction, to read

$$
\begin{aligned}
x_{y p}(y, p)(r)(q)= & -A(y, p)^{-1} D^{-}\left\{h_{x x}[x(y, p), p]\left[x_{p}(y, p) q\right]\left[x_{y}(y, p) r\right]\right. \\
& \left.+h_{x p}[x(y, p), p]\left[x_{y}(y, p) r\right][q]\right\} .
\end{aligned}
$$

2. Eq. (3.7) should read

$$
\operatorname{minimize}\left\{\left\langle\phi_{y}\left(0, p_{0}\right)-w, y\right\rangle+\frac{1}{2}\left\langle y, \phi_{y y}\left(0, p_{0}\right) y\right\rangle \mid y \in T_{0} \cap K\right\} .
$$

